\title{
Minimally Invasive Vacuum-Assisted Closure Therapy With Instillation (Mini-VAC-Instill) for Pleural Empyema
}

\author{
Hans-Stefan Hofmann, MD ${ }^{1,2}$, Reiner Neu, MD', Tobias Potzger, MD², \\ Rudolf Schemm, MD', Christian Grosser', Tamas Szöke, MD', \\ and Zsolt Sziklavari, MD'
}

\begin{abstract}
Enthusiasm for minimally invasive thoracic surgery is increasing. Thoracoscopy plays a significant therapeutic role in the fibrinopurulent stage (stage II) of empyema, in which loculated fluid cannot often be adequately drained by chest tube alone. For some debilitated and septic patients, further procedures such as open-window thoracostomy (OWT) with daily wound care or vacuum-assisted closure (VAC) therapy are necessary. In the present article, we propose a new option of minimally invasive VAC therapy including a topical solution of the empyema without open-window thoracostomy (Mini-VAC-instill). Three patients who underwent surgery using this technique are also presented. The discussion is focused on the advantages and disadvantages of the approach.
\end{abstract}

\section{Keywords}

pleural empyema, VAC-instill, minimally invasive thoracic surgery

\section{Introduction}

Despite significant advances in the treatment of thoracic infections, pleural empyema remains a problem in modern thoracic surgery. If the patient is medically unstable, the evacuation of pus and debridement can be managed by open-window thoracostomy (OWT) ${ }^{1,2}$ Vacuumassisted closure (VAC) therapy supports the treatment of acute or chronic wound infections both in general and in thoracic surgery. These benefits include the removal of accumulating fluid, increased oxygen tension in the wound, increased blood flow, and increased granulation tissue proliferation. The combination of OWT and VAC therapy should be discussed as soon as possible, especially for complicated pleural empyema and in patients with an increased risk for impaired wound healing. ${ }^{3}$ The Mini-VAC procedure offers a minimally invasive approach with the abdication of an OWT. ${ }^{4}$

In cases of highly infected/bacterial colonised pleural empyemas, flushing by a chest tube or the OWT is often necessary. In this report, we demonstrate that the MiniVAC treatment concept can also be used for the intermittent instillation of the pleural cavity through the VAC sponge (Mini-VAC-instill).

\section{Technique}

Under general anaesthesia, a 5- to 6-cm long incision centred over the area of the greatest pleural fluid collection was made (Figure 1A). The intercostal muscle was divided, and the empyemal cavity was opened. After the intercostal aspiration of all pus and necrotic debris, the cavity was flushed with a polyhexanide solution (Lavanid $0.02 \%$, Serag-Wiessner KG, Naila, Germany). Then, the VAC sponge (Instill Dressings, KCI Medical, Wiesbaden, Germany; 400-600 $\mu \mathrm{m}$ ) was introduced in the pleural cavity over a flexible polymer membrane (Alexis, Applied Medical, Rancho Santa Margarita, CA) retractor (technique described earlier ${ }^{4}$; Figure 1B and C). On the top of the VAC sponge, the VAC instill pad, including the tubing, was positioned (Figure 1D). The

\footnotetext{
'Hospital Barmherzige Brüder Regensburg, Regensburg, Germany

${ }^{2}$ University Regensburg, Regensburg, Germany

Corresponding Author:

Hans-Stefan Hofmann, Department of Thoracic Surgery, Hospital

Barmherzige Brüder Regensburg, Prüfeningerstraße 86, 93049

Regensburg, Germany.

Email: hans-stefan.hofmann@barmherzige-regensburg.de
} 


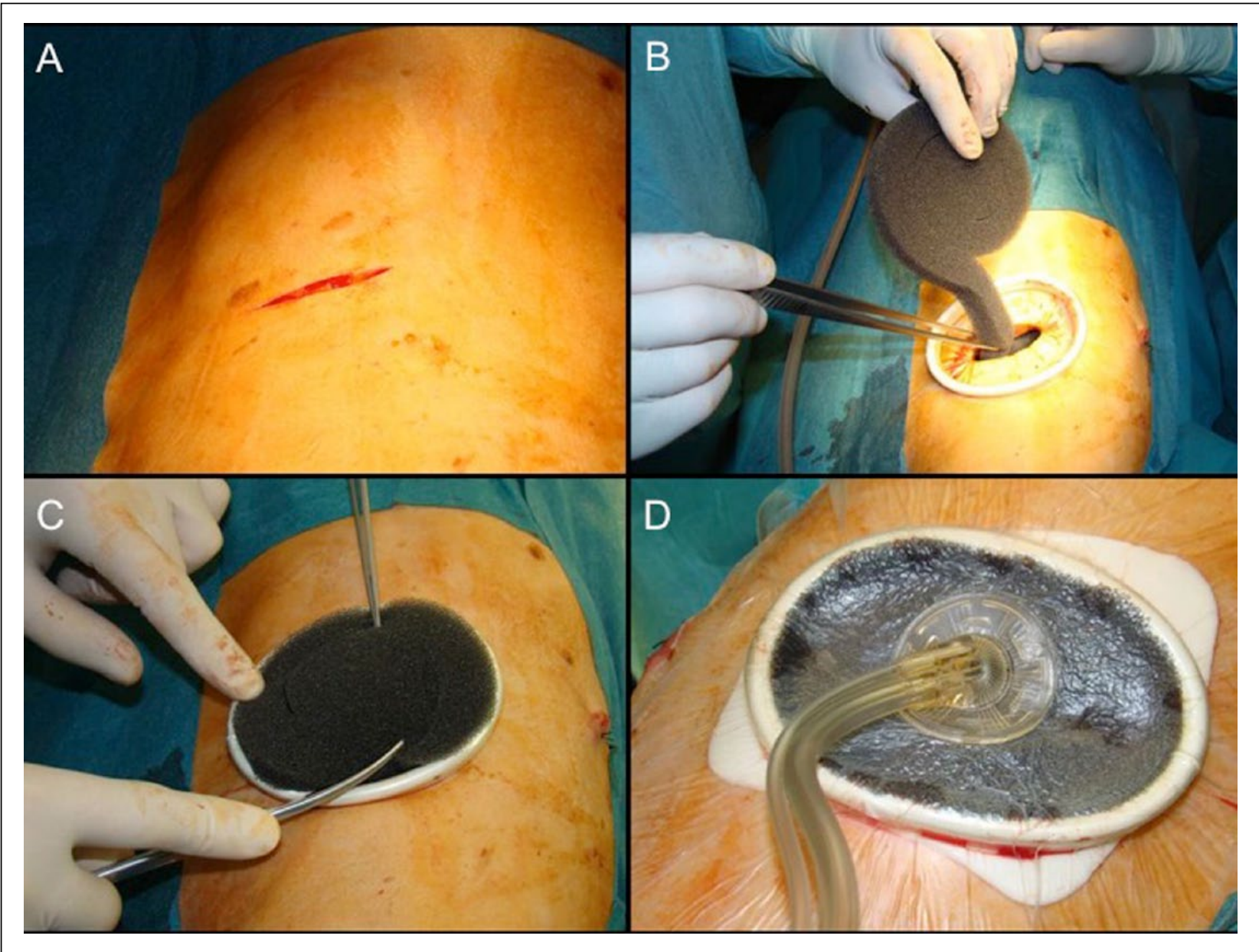

Figure I. Technique of Mini-VAC-instill procedure: $5-\mathrm{cm}$ incision over the pleural empyema (A), introduction of VAC sponge over ALEXIS retractor $(B, C)$, and VAC-instill pad (D). VAC, vacuum-assisted closure.

wound volume determines the amount of fluid instilled. The vacuum device coupled with automated, controlled antiseptic fluid delivery regulates the instilled fluid volume. The volume of the pleural space was tested and determined (approximately $150 \mathrm{~mL}$ ). The level of suction was set to $-100 \mathrm{~mm} \mathrm{Hg}$ from the start. The flushing of the pleural cavity was performed with a predetermined amount of $0.02 \%$ polyhexanide solution (Lavanid) in an automated topical solution delivery modus (30 minutes every 8 hours). The patients were treated in parallel with wide spectrum antibiotics. Based on the microbiological results, the spectrum was occasionally modified. The Mini-VAC-instill system was changed every 3 to 4 days. Only a small amount of debridement was required at each sponge change. The remaining pleural space was, in all cases after the MiniVAC-instill therapy, very small and only filled with an aminoglycoside antibiotic gentamicin sponge (GentaColl resorb, Resorba GmbH, Nürnberg, Germany).

\section{Case Reports}

The demographic, clinical, and treatment characteristics of the patients are given in Table 1 .

\section{Case I}

A 56-year-old man was diagnosed with pleural empyema after a third course of palliative chemotherapy for gastric cancer stage IV. The pleural space was highly colonized with Escherichia coli and Bacillus cereus. The decision for quick and complete eradication was made. Because of the poor general condition of the patient (Karnofsky index $50 \%$ ), we rejected an extensive surgical procedure (decortication). Considering the high bacterial colonization, the Mini-VAC-instill system was implemented. After eleven days of treatment, the pleural cavity was visually determined to be clean, and the patient had 2 negative quantitative cultures. On the 12th postoperative 
Table I. Demographic, Clinical, and Treatment Characteristics.

\begin{tabular}{llll}
\hline Characteristic & Patient I & Patient 2 & Patient 3 \\
\hline Age (years)/gender & $75 /$ male & $57 /$ male & $87 /$ female \\
Karnofsky index & 50 & 50 & 40 \\
Multimorbidity & Yes & No & No \\
Immunosuppression & No & Yes (chemotherapy) & No \\
Etiology & Primary & Primary & Secondary \\
Empyema & Stage II & Stage II & Stage II \\
Primary intervention & Chest tube & Chest tube & Chest tube \\
Microbiology & Escherichia coli & Escherichia coli, Bacillus cereus & Staphylococcus aureus \\
Mini-VAC-instill therapy (days) & 5 & I2 & I0 \\
VAC-changes & I & 3 & I \\
Microbiology after Mini-VAC-instill & Sterile & Sterile & Sterile \\
Discharge after Mini-VAC-instill & 5th day & 4th day & 7 day \\
\hline
\end{tabular}

${ }^{\text {a }}$ More than or equal to 4 chronic diseases.

day, the remaining pleural space was filled with a haemostyptic collagen sponge containing the aminoglycoside antibiotic gentamicin (Genta-Coll resorb). The wound was closed with single stitches and healed per primam. The chemotherapeutic treatment of the gastric tumor was continued after 2 weeks.

\section{Case 2}

A 75-year-old male patient with a Karnofsky index of 50\% presented to our department with recurrent fever that reached as high as $38^{\circ} \mathrm{C}$ and chills that had lasted for 1 month. He also reported cough, dyspnea, and expectoration. He was an active smoker with a 40-pack-year history of smoking. Routine laboratory testing showed inflammation. The chest X-ray showed a pleural effusion, and the chest computed tomography (CT) raised the suspicion of pleural empyema stage II. The patient underwent a tube thoracostomy. Escherichia coli and anaerobic species were isolated. The primary drainage treatment failed despite daily solution changes. The decision to use the Mini-VACinstill was made. The VAC sponge was changed only once. After 5 days of the Mini-VAC-instill treatment with $0.02 \%$ polyhexanide, the pleural cavity was sterile and could be primarily closed. The patient was discharged from the hospital on the fifth postoperative day. At the 1-month followup, no pleural empyema had recurred.

\section{Case 3}

A 87-year-old woman presented with left inspiratory chest pain in the emergency room. The medical history revealed a left thoracotomy for a kyphoplasty of thoracic vertebra 12 and decompression of the spinal canal three months earlier. In the past 6 weeks, she suffered with a fever and significant worsening. The physical examination demonstrated cachexia of the patient, and auscultation and percussion of the lungs revealed fluid in the left hemithorax. Her arterial oxygen saturation at rest while breathing room air was $90 \%$, and her C-reactive protein level was increased by more than 100-fold. The CT scan of the thorax showed a fracture of thoracic vertebra 8 and both an intraspinal and stage II pleural empyema (Figure 2A). Antibiotic therapy with piperacillin/sulbactam was started. The microbiological diagnosis was Staphylococcus aureus. After a chest tube thoracostomy, thoracic vertebra 8 was dorsally stabilized by the neurosurgeons. Pus was demonstrated in the chest tube and in the intraspinal region. The chest tube was flushed with betaisodona $10 \%$ thrice per day. Because this treatment failed clinically and on the CT scan control, the decision to use the Mini-VAC-Instill was made 14 days after the neurosurgical operation. The Mini-VAC-instill therapy was performed with $150 \mathrm{~mL}$ of $0.02 \%$ polyhexanide solution, and the VAC sponge was only changed once. The pleural cavity was sterile and could be primarily closed on the seventh day after the first installation of the Mini-VAC. She was discharged from the hospital 5 days after primary chest closure. After the follow-up at 1 month, no pleural empyema had recurred (Figure 2B).

\section{Discussion}

The VAC-instill therapy differs from the standard VAC treatment in that topical solutions are cyclically flushed into the foam dressing and held for a user-selected period before removal under negative pressure. Studies have reported on the effectiveness of VAC-instill in wounds with high levels of exudate and pus and in acute traumatic wounds or wounds acutely debrided due to infected soft tissue. ${ }^{5,6}$

The bacteriology of pleural empyemas is often polymicrobial and mixed, containing multiple species of both 


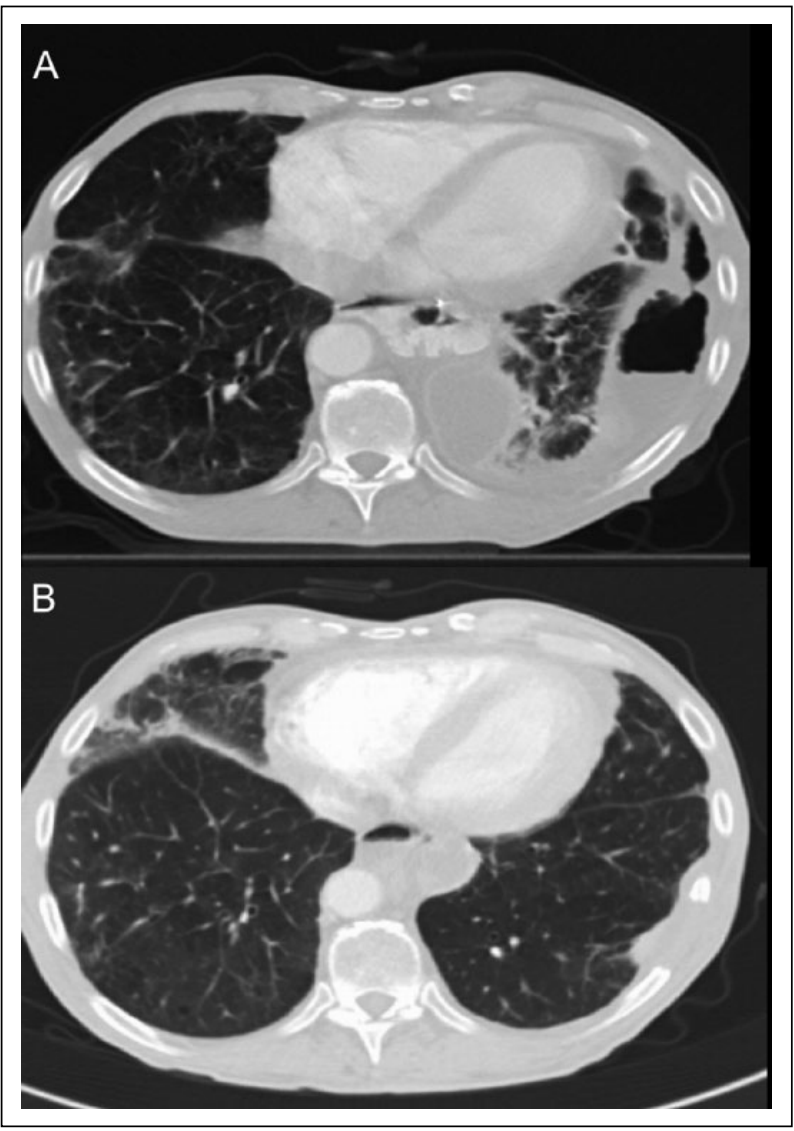

Figure 2. Chest CT scan of pleural empyema before $(A)$ and after (B) Mini-VAC-instill therapy. CT, computed tomography; VAC, vacuum-assisted closure.

aerobic and anaerobic bacteria, with the latter found in up to $75 \%$ of the cases. ${ }^{7}$ The diffusion of antibiotics into the pleural fluid is good, but certain agents may be inactivated in the presence of pus, low $\mathrm{pH}$, and beta-lactamase enzymes. In addition to a surgical intervention, flushing of the pleural cavity by a chest tube is often necessary to clean the empyemal cavity. If the patient is also medically unstable, the evacuation of pus and debridement can be managed traditionally by OWT and daily open wound treatment, including flushing of the pleural space.

The present Mini-VAC-instill therapy (an upgrade of Mini-VAC) guarantees the advantage of an open treatment, including flushing without OWT. Rib resection for OWT is associated with more postoperative pain and a muscle flap closure in most cases.

The decision not to proceed with classical decortication of the lung appears not to be a disadvantage because similar lung reexpansion was achieved by debridement alone without decortication in patients presenting with empyema. ${ }^{8}$

The Mini-VAC-instill allows pleural cleaning, irrigation and removal of infectious materials. Solutions intended for topical use include topical cleansers, antibiotics, antifungals, and antiseptics. Intervals in pleural flushing (the ebb-and-flow technique) are better than continuous pleural flushing with standard intervals. ${ }^{9}$ In practice, these cycles of flushing are repeated from 4 to 12 times hourly. ${ }^{10,11}$ According to the present state of knowledge, polyhexanide is the first-choice antiseptic solution when using instillation for acutely or chronically infected wounds (after debridement). ${ }^{12}$ However, the key aspect of this therapy is the delivery method of the topical solution, not the type of solution.

The system should only be exchanged every 3 to 4 days. Thus, daily time-consuming doctor and caregiver wound intervention is not necessary. The sponge changes are facile and fast because the Mini-Vac-instill therapy also enables minimization of intraoperative manual activities (eg, debridement) by fast cleaning.

The wound should be assessed at every dressing change, and the decision to continue the Mini-VAC-instill should be made during this time. We recommend Mini-VAC-instill therapy only for hospital use because patients are often septic and infected by multiple species of bacteria. After cleaning of the pleural space, conversion to simple Mini-VAC therapy to bridge to the ambulant sector, with the aim of further reduction of the pleural cavity, is possible.

\section{Conclusions}

Standard pleural VAC therapy offers rapid treatment for complex pleural empyema. In septic patients with highly infected/bacterially colonized pleural empyema, we recommend Mini-VAC-instill therapy. This procedure provides intermittent topical solution delivery and removal from the wound site. Wound cleaning, the removal of infectious material and wound healing are supported by this system. The procedure is minimally invasive and highly compatible, especially in patients in poor general condition.

\section{Declaration of Conflict Interests}

The author(s) declared no conflicts of interest with respect to research, authorship, and/or publication of this article.

\section{Funding}

The author(s) received no financial support for the research, authorship, and/or publication of this article.

\section{References}

1. Eloesser L. An operation for tuberculous empyema. Chest. 1935;1(8):8-9, 23.

2. Clagett OT, Geraci JE. A procedure for the management of postpneumonectomy empyema. J Thorac Cardiovasc Surg. 1963;45:141-145. 
3. Sziklavari Z, Grosser C, Neu R, et al. Complex pleural empyema can be safely treated with vacuum-assisted closure. J Cardiothorac Surg. 2011;6:130.

4. Hofmann HS, Schemm R, Grosser C, Szöke T, Sziklavari Z. Vacuum-assisted closure of pleural empyema without classic open-window thoracostomy. Ann Thorac Surg. 2012;93:1741-1742.

5. Gabriel A, Shores J, Heinrich C, et al. Negative pressure wound therapy with instillation: a pilot study describing a new method for treating infected wounds. Int Wound J. 2008;5:399-413.

6. Gabriel A, Shores J, Bernstein B, et al. A clinical review of infected wound treatment with vacuum assisted closure (V.A.C.) therapy: experience and case series. Int Wound J. 2009;6(suppl 2):1-25.

7. Hughes CE, Van Scoy RE. Antibiotic therapy of pleural empyema. Semin Respir Infect. 1991;6:94-102.
8. Kho P, Karunanantham J, Leung M, Lim E. Debridement alone without decortication can achieve lung re-expansion in patients with empyema: an observational study. Interact Cardiovasc Thorac Surg. 2011;12:724-727.

9. Engelmann C. Septische Thoraxchirurgie. Berlin, Germany: Pabst Science; 1998.

10. Rosenfeldt FL, McGibney D, Braimbridge MV, Watson DA. Comparison between irrigation and conventional treatment for empyema and pneumonectomy space infection. Thorax. 1981;36:272-277.

11. Conlan AA, Abramor E, Delikaris P, Hurwitz SS. Taurolidine instillation as therapy for empyema thoracis. A prospective study of 50 patients. S Afr Med J. 1983;64:653-655.

12. Kramer A, Assadian O, Below H, Willy C. Wound antiseptics today - an overwiew. In: Willy C, ed. Antiseptics in Surgery-Update 2013. Berlin, Germany: Lindqvist Books; 2013:83-110. 